\title{
Mutual Prodrugs of Piroxicam
}

\author{
Mohd Imran ${ }^{1}$ and Mohammad Asif ${ }^{2 *}$ \\ ${ }^{1}$ Department of Pharmaceutical Chemistry, Faculty of Pharmacy, Northern Border University, Rafha, Saudi Arabia \\ ${ }^{2}$ Department of Pharmaceutical Chemistry, Himalayan Institute of Pharmacy Research, Dehradun, India
}

*Corresponding Author: Mohammad Asif, Professor, Department of Pharmaceutical chemistry, Himalayan Institute of Pharmacy Research, Dehradun, India.

Received: May 20, 2019; Published: June 07, 2019

DOI: $10.31080 /$ ASPS.2019.03.0305

Most of the drug molecules in present use are linked with some intolerable physicochemical or biopharmaceutical factors. Nonsteroidal anti-inflammatory drugs (NSAIDs) have possibility of common adverse effects like dyspepsia or acidity and ulceration that limits their uses [1]. These NSAIDs are commonly used in the treatment of pain and inflammation in various conditions like osteo/rheumatoid arthritis (OA/RA) [2]. Progression in the side effects of gastrointestinal tract (GIT) mainly stomach ulceration, bleeding and damage of stomach mucus membrane are the main limitation in use. This is due to local effect applied by direct contact of drug with gastric mucosa [3]. The enolic hydroxyl group of piroxicam and free acidic group of aryl propionic acids plays a key role in sustaining the efficiency and making the gastric ulceration as well [4]. There are several advances reported that are used to overcome the side effects of NSAIDs by chemical derivatization with retention of effectiveness. Mutual prodrug concept involves the conjugation of two biologically active drugs in which individual drug acts as promoiety. This concept has used for reduced GI toxicity by temporarily masking enolic group of piroxicam with acidic group of other NDAIDs drugs and also enhance their absorption5. Prodrugs of piroxicam is of intense attention for medicinal chemist as the enolic hydroxyl group can be derivatized simply and could results in expanded derivatives like ampiroxicam, one of the extensively used drug [6-8]. Several prodrugs of selected NSAIDs were also reported $[9,10]$. A new series of mutual prodrugs of piroxicam is designed by combine it with well-known NSAIDs like aceclofenac, ibuprofen, mefenamic acid and naproxen as active promoieties. The enolic hydroxyl group of piroxicam is conjugated with acidic group of propionic acid derivatives to get esters.

\section{Synthesis of piroxicam prodrugs}

Mutual prodrug ester derivatives, piroxicam-aceclofenac ester (PA), piroxicam-ibuprofen ester (PI), piroxicam-mefenamic acid ester (PM) and piroxicamnaproxen ester (PN) (Figure 1) were synthesized by first converting the promoieties having acidic group into corresponding acid chlorides and followed by reaction with hydroxyl group of piroxicam (Scheme 1).<smiles>CC1C(C(=O)Nc2ccccn2)=C(OC(=O)COC(=O)Cc2ccccc2Nc2c(Cl)cccc2Cl)C2=CCCCC2S1(=O)=O</smiles>

Picroxicam+Acceclofenac (PA)

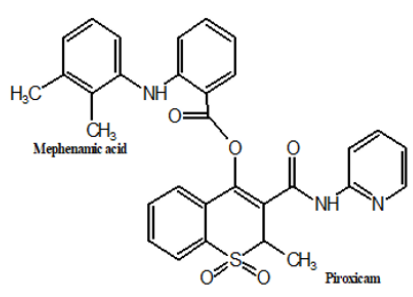

Picroxicam+Mephenamic acid (PM)<smiles>CC(C)c1ccc(C(C)C(=O)OC2=C(C(O)Nc3ccccn3)C(C)S(=O)(=O)[C@@H]3CCCC[C@H]23)cc1</smiles>

Picroxicam+Ibuprofen|(PI)

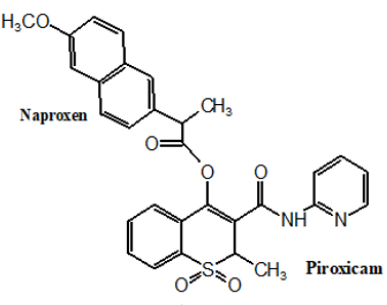

Picroxicam+Naproxen $\mid$ (PN)
Figure 1: Structure of mutual prodrugs of Piroxicam.

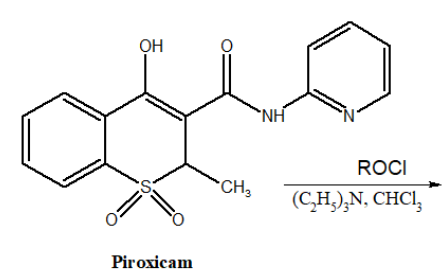

Piroxicam

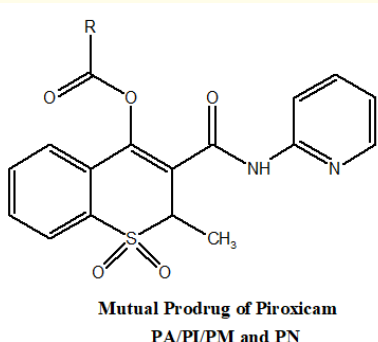

PA/PI/PM and PN
Scheme 1: RCOOH=NSAIDs (Aceclofenac/Ibuprofen/ Mefenamic acid/ Naproxen). 


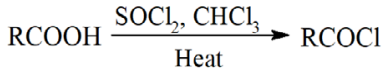

This approach in overcoming the disagreeable properties of accessible drugs by designing the mutual prodrugs. The synthesis of mutual prodrugs of piroxicam was get with well-known NSAIDs. The targeted prodrugs PA/PI/PM and PN (Figure 1) were formed (Scheme 1) successfully by altering the selected NSAIDs (aceclofenac, ibuprofen, mefenamic acid and naproxen) to their own acid chlorides by using thionyl chloride. These NSAIDs act as masking agent for enolic hydroxyl group of piroxicam. The acid chlorides were then coupled with piroxicam through enolic hydroxyl in presence of chloroform and triethylamine. The necessary precondition for success in the use of prodrugs is that the masked drugs should be acid stable to avoid the direct contact effects with the gastric mucosa as well as the local inhibition of the prostaglandins (PGs) [11]. These piroxicam prodrug derivatives were expected across the suitable clinical range having acidic, neutral and alkaline $\mathrm{pH}$ values. The hydrolysis kinetics result showed that prodrugs of piroxicam undergo chemical degradation with first order kinetics, and consequently quantitatively changed to parent drug. All these prodrugs showed high stability in acidic condition; this indicates that the drugs passed unhydrolyzed through the stomach on oral use [12]. The degradation capacity of prodrugs at neutral and alkaline $\mathrm{pH}$ is an indication of their susceptibility for hydrolysis.

The mutual prodrug approach can be successfully applied in attaining the goal of enhancing the therapeutic values of piroxicam; masking of enolic hydroxyl group through acids and converting them to esters and using the NSAIDs for getting the synergistic effects (anti-inflammatory and gastro-protective effects). Mutual prodrug approach gives an opportunity to medicinal chemist for improving the clinical and therapeutic values of a drug that is suffering from unwanted properties hampering its clinical values.

\section{Acknowledgements}

Authors are thankful to Himalayan Institute of Pharmacy and Research (India), for providing technical support and facilities.

\section{Bibliography}

1. Pal S., et al. "Synthesis and structure analysis of cyclodehydration product of piroxicam: A metabolite detected in dogs and monkeys". European Journal of Medicinal Chemistry 44 (2009): 3368-3371.

2. Kawai S., et al. "Recent advances in nonsteroidal anti-inflammatory drugs". Allergology International 54.2 (2005): 209-215.
3. Kean WF and Buchanan WW. "The use of NSAIDs in rheumatic disorders 2005: a global perspective". Inflammopharmacology 13.4 (2005): 343-370.

4. Redasani VK., et al. "Synthesis and evaluation of novel mutual prodrugs of Piroxicam". Journal of Pharmaceutical Chemistry 1.1 (2017): 1-4.

5. Bhosle D., et al. "Mutual Prodrug Concept: F Mutual Prodrug Concept: Fundamentals and undamentals and Applications". Indian Journal of Pharmaceutical Sciences (2006): 286.

6. Jayaselli J., et al. "Derivatization of enolic $\mathrm{OH}$ of piroxicam: a comparative study on esters and sulfonates". Journal of Brazilian Chemical Society 19.3 (2008): 509-515.

7. Redasani VK., et al. "Antiinflammatory and gastroprotective evaluation of prodrugs of piroxicam". Ulcers (2014).

8. Carty TJ., et al. "Ampiroxicam, an anti-inflammatory agent which is a prodrug of piroxicam". Inflammation Research 39.3 (1993): 157-165.

9. Mahfouz NM., et al. "Cyclic amide derivatives as potential prodrugs II: N-hydroxymethylsuccinimide-/isatin esters of some NSAIDs as prodrugs with an improved therapeutic index". European Journal of Medicinal Chemistry 34.7 (1999): 551-562.

10. Redasani VK and Bari SB. "Synthesis and evaluation of mutual prodrugs of ibuprofen with menthol, thymol and eugenol". European Journal of Medicinal Chemistry 56 (2012): 134-138.

11. Omar FA. "Cyclic amide derivatives as potential prodrugs. Synthesis and evaluation of N-hydroxymethylphthalimide esters of some non-steroidal anti-inflammatory carboxylic acid drugs". European Journal of Medicinal Chemistry 33.2 (1998): 123-131.

12. Redasani VK and Bari SB. "Synthesis and evaluation of glyceride prodrugs of naproxen". Open Journal of Medicinal Chemistry 3 (2013): 87-92.

Volume 3 Issue 7 July 2019

(C) All rights are reserved by Mohd Imran and Mohammad Asif. 